\title{
L'EXPÉRIENCE DU DEUIL CHEZ LES MIGRANTS CENTRAMÉRICAINS EN TRANSIT AU MEXIQUE
}

\section{EXPERIENCES OF GRIEF AMONG CENTRAL AMERICAN MigRANTS IN TRANSIT THROUGH MEXICO ${ }^{1}$}

\section{Philippe Stoesslé}

Département de sciences sociales, Universidad de Monterrey, philippe.stoessle@udem.edu

\author{
Ana Victoria Rodriguez-Maroun \\ Gestionnaire de programme, Center for International Policy, Mexico, \\ ana.rodriguez.m22@gmail.com
}

\begin{abstract}
Résumé / Abstract
Le Mexique est un point de passage obligé pour les migrants centraméricains en situation irrégulière qui tentent d'atteindre les États-Unis. En intégrant des contributions de la psychologie sociale et de la sociologie, notre étude exploratoire se concentre sur un sousgroupe extrêmement vulnérable de cette population. Nous avons mené dix entretiens en profondeur avec des migrants qui ont vécu le deuil d'un être cher du premier cercle familial pendant leur transit au Mexique. Après avoir analysé leurs stratégies d'adaptation au deuil et l'importance des réseaux de soutien social, nous avons observé une association entre le capital social des migrants et leur capacité de résilience. Ce travail fournit des informations qui peuvent servir de base pour de plus amples recherches liées au thème de la mort en contexte de mobilité.
\end{abstract}

Mexico has become a crucial crossing point for migratory flows of Central American migrants who use it as a way to enter the United States. Integrating contributions from social psychology and sociology, this study focuses on a sector of this population that is in a situation of extreme vulnerability. Ten in-depth interviews were carried out with migrants who had to deal with grief as a result of the loss of a loved one within the first family circle while in transit through Mexico. After analyzing their coping strategies to grief and the importance of social support networks, it was observed that there is an association between the migrant's social capital and his or her capacity for resilience. This study offers empirical evidence that can serve as a basis for further investigations related to the issue of death and migration.

Mots clés : Immigration irrégulière, mort, deuil, réseaux, Mexique.

Keywords: Irregular migration, death, grief, networks, Mexico.

\footnotetext{
${ }^{1}$ Article disponible dans sa version finale sur Érudit : https://www.erudit.org/fr/revues/du/2018-v18-du04559/1059594ar/
} 


\section{Introduction $^{1}$}

Pertes et deuils, éléments intrinsèques de l'expérience migratoire

Toute migration constitue une « rupture vitale » (Bar de Jones 2001 : 10). L’acte d'émigrer, plus qu'un simple déplacement humain, suppose un processus qui renferme des espoirs et des illusions personnelles et collectives (Estrada 2012), mais qui génère aussi des incertitudes, des peurs, des angoisses, de la dépression et de l’anxiété (Achotegui 2009).

De nombreuses études montrent les effets traumatisants de la migration sur les personnes qui vivent le deuil de leur pays, lorsque se produit une tension entre un passé angoissant géographiquement lointain et un futur supposément meilleur, bien que, par définition, incertain (Akhtar 1999, cité par Kogan 2010; Bertrand 1990; Casado et Leung 2001; Grinberg et Grinberg 1986).

Joseba Achotegui $(2009,2004)$ regroupe en sept catégories de «deuils migratoires » les multiples pertes symboliques et physiques, temporelles ou permanentes qu'affrontent les migrants à partir du moment où ils partent de chez eux : le deuil de la famille et des êtres chers, de la langue, de la culture, de la terre, du statut social, du contact avec le groupe national et de l’intégrité physique. Le deuil lié à la mort d’un être cher est probablement le plus douloureux, car il est irréversible (Achotegui 2004).

\section{Le deuil pendant le parcours migratoire}

Cette étude exploratoire aborde le thème du deuil lié à la perte d'un parent proche chez les migrants centraméricains en situation irrégulière au Mexique et en transit vers les États-Unis. 
Notre objectif est de relever les principaux facteurs de protection et de risque associés au deuil et de discuter du rôle des stratégies observées et des réseaux de soutien social dans ce processus.

Les trajectoires migratoires s’inscrivent dans un contexte d'extrême vulnérabilité. Aussi, une connaissance approfondie des ressources et des comportements sociaux construits par les migrants endeuillés durant leur transit par le Mexique pourra fournir les informations nécessaires au développement et à l'amélioration des pratiques d'accompagnement offertes par les organisations de la société civile et les autorités locales ou nationales.

En effet, l'adaptation des personnes, au cours du processus migratoire, à la perte d'un parent proche constitue un phénomène complexe et peu étudié. Il existe des travaux dans plusieurs disciplines et dans plusieurs pays sur le deuil des immigrés, soit ceux qui sont arrivés à leur destination (Chéron-Leboeuf et al. 2016; De la Revilla et al. 2011; Falicov 1999; Rachédi et al. 2016). Cependant, il y en a peu sur les personnes migrantes qui perdent un membre de leur famille pendant leur parcours migratoire, et encore moins sur les migrants irréguliers qui vivent dans un contexte de forte marginalisation sociale liée à leur situation migratoire.

Ce voyage dure plusieurs semaines, voire des mois, et la perte s'effectue dans un nouvel espace culturel (le Mexique), où les migrants irréguliers affrontent un contexte de violence, de pénurie de ressources économiques et de manque d'aide médico-sociale et juridique (Comisión Nacional de los Derechos Humanos 2011; Fernández de Castro 2012; Infante et al. 2012; Kuhner 2011; Mejía et Cruz 2014; Médicos Sin Fronteras 2014; Organisation internationale pour les migrations 2010). Ce « processus inclut des niveaux de stress tellement intenses qu'ils dépassent la capacité d’adaptation des êtres humains » (Achotegui 2004 : 46, notre traduction), créant une perception intense et abrupte de la perte de l'être cher. Le peu de possibilités 
d'affronter les circonstances qui émanent de la nouvelle configuration familiale après la mort du parent proche (comme la disparition d'une source de revenus ou le besoin de travailler pour maintenir sa famille restée seule) avive la nécessité pour les migrants en transit de chercher des réseaux de soutien (qu’ils soient gouvernementaux, religieux ou civils).

Dans ces conditions, le processus du deuil est logiquement plus complexe et contient plus de souffrances que dans le cas d'un deuil régulier, en raison de la désorientation culturelle que peut éprouver l’endeuillé. Comme l’affirme Cabodevilla (2007 : 165, notre traduction) : « La manière dont nous comprenons le processus de deuil est liée à la manière dont nous nous comportons face à la mort dans le milieu culturel auquel nous appartenons. »

En effet, la clandestinité dans laquelle vivent ces migrants renforce la dépendance qu'ils ont envers les réseaux d'aide et d'entraide, sans lesquels les conséquences des troubles psychosociaux dont ils souffrent seraient potentiellement destructrices. Très souvent, l'abus d'alcool, de tabac et de substances constitue un recours pour surmonter la solitude, l'éloignement des repères familiaux et les situations traumatisantes que l'angoisse de la perte d'un être cher en contexte de mobilité peut encore renforcer (Bennegadi 1984; Bhugra et Bhui 2018; Michel 2015). 


\section{Cadre méthodologique}

\section{Les participants}

La présente étude a été approuvée par l’Universidad de Monterrey (Mexique) dans le cadre du projet UIN15012 « Accès à la santé des migrants en transit au Mexique ». Nous nous sommes entretenus avec dix personnes migrantes originaires d’Amérique centrale, toutes en situation irrégulière et ayant plus de 18 ans. Les entretiens se sont déroulés de juillet à octobre 2016 dans deux refuges (Casas del migrante) ${ }^{2}$ : Hermanos en el Camino, situé dans la ville de Ciudad Ixtepec, au sud du Mexique, et Casanicolás, de Guadalupe, au nord-est du pays. Ces dix personnes vivaient toutes un deuil lié à la perte d'un être cher membre de leur famille. Pour les participants le temps écoulé depuis le décès variait entre 7 et 459 jours, avec une durée moyenne de 77 jours. Le tableau 1 présente le profil sociodémographique des participants.

Tableau 1. Profil sociodémographique des participants

\begin{tabular}{lc} 
Variable & Fréquence \\
\hline Sexe & \\
\hline Femmes & 3 \\
Hommes & 7 \\
Âge & 18-42 $(30,2)$ \\
\hline Religion & 5 \\
\hline Catholique & 3 \\
Protestant & 1 \\
" Sainte Mort ${ }^{3}{ }^{\text {» }}$ & 1 \\
Indéfinie & \\
Pays d'origine & 6 \\
\hline Honduras & 4 \\
Salvador & \\
État civil & 5 \\
\hline Célibataire & 3 \\
Union libre & 1 \\
Marié & 1 \\
Marié et union libre &
\end{tabular}




\section{Les motifs et les trajectoires}

La migration irrégulière est un phénomène complexe dont les déterminants sont nombreux et échappent parfois au discours des personnes migrantes. Nous nous contenterons de présenter ici les motifs d’émigration évoqués lors des entretiens. Bien que les dix sujets interviewés soient originaires de pays différents, le principal point commun entre eux est la violence qui sévit dans leur pays d’origine et qui a été signalée comme principal motif d’émigration (dans neuf cas sur dix). Par ailleurs, deux participants ont mentionné l'extrême pauvreté en plus de l’insécurité généralisée. Seul un participant a quitté son pays de manière volontaire, pour trouver de meilleures possibilités ailleurs, même si son récit faisait constamment référence à la violence qui règne en Amérique centrale ${ }^{4}$.

Ainsi, toutes les personnes interrogées ont relaté une trajectoire de vie caractérisée par la violence structurelle, par la souffrance et par la douleur; leur existence est marquée par un environnement mortifère depuis leur plus jeune âge. Cette violence objective imprègne aujourd'hui leurs relations sociales et familiales. Elle s'explique aisément par les guerres civiles qui sévissent dans la région, laissant de nombreuses personnes sans emploi et donnant un accès facile aux armes. Ceci se reflète dans les témoignages qui font référence aux Maras. En effet, tous les participants ont vécu des épisodes d'extorsion de leurs biens par les Maras ou ont perdu des parents et des amis assassinés par ces gangs.

Les Maras sont des groupes de dizaines de milliers de personnes impliquées dans des activités criminelles telles que le trafic de drogue ou l'extorsion : des petites entreprises et des résidents des zones qu'ils contrôlent, des compagnies de transports, des vendeurs ambulants, des personnes qui reçoivent de l'argent de l'étranger ou encore de personnes qui manquent de loyauté, selon la Mara. Les Maras imposent à la population des codes tacites de conduite. Les 
personnes peuvent voir, écouter, mais ne peuvent jamais dénoncer ce qui se passe ou en parler, au risque d’être torturées ou assassinées (Jimenez 2016).

\section{Les procédures d'entretien et la méthode d'analyse}

La situation de deuil des participants a été identifiée par les employés des refuges lors de l'entretien de bienvenue auquel toutes les personnes doivent se soumettre à leur arrivée. Ils ont été approchés le jour suivant et invités à participer à un entretien en profondeur de 90 à 150 minutes. Cet instrument nous a permis d'explorer en détail les perceptions et les représentations des migrants par rapport au deuil, ainsi que de comprendre leur vision du monde et les dilemmes vécus lors de leurs expériences migratoires.

Tous ont accepté volontairement et de manière éclairée de répondre à nos questions dans les espaces communs des refuges (salle à manger, jardin, cuisine, salle de jeux), après avoir été instruits sur l'importance de leur participation, la garantie du respect de leur anonymat, la confidentialité de l'étude et la possibilité de s’en retirer à tout moment.

Les questions portaient sur leur profil sociodémographique et sur leur histoire familiale, sur les motifs de leur émigration, leurs expériences positives et négatives de voyage dans un nouvel espace culturel (spécialement en relation avec les « sept deuils symboliques » d’Achotegui), leur expérience du deuil (type de mort, annonce, contacts avec la famille, stratégies de résilience, réajustement du projet migratoire, religiosité et pratiques spirituelles), sur leur gestion du stress, le rôle des différents acteurs (en intégrant le niveau de confiance et la fréquence des contacts), sur les soutiens sociaux dont ils ont bénéficié et sur la manière dont ils ont utilisé l'aide reçue. 
Les entretiens ont été transcrits, codés et analysés pour définir les catégories d’analyse présentées ci-dessous. Par cette démarche, nous avons dégagé un sens des récits des participants et identifié les ressources construites au cours de leur périple.

\section{Résultats et discussion}

Pour comprendre l'expérience du deuil des migrants centraméricains en transit au Mexique, nous avons fondé notre analyse sur les trois dimensions du deuil qu'identifient Stroebe et Schut (2001) dans leur livre Handbook of Bereavement Research: Consequences, Coping and Care : le type de décès, les facteurs individuels et les facteurs interpersonnels.

La première dimension fait référence aux conditions de la mort, selon qu'elle est subite ou anticipée, par exemple dans le cas d'une maladie, mais aussi au lien et à la proximité généalogique avec le défunt. La deuxième dimension concerne les facteurs individuels, qui incluent l’intégration préalable à un groupe social (amical, religieux, sportif ou professionnel, entre autres), la stabilité émotionnelle de la personne, ses croyances religieuses, ses caractéristiques sociodémographiques et ses expériences avec les institutions dans le pays de transit. Enfin, la dernière dimension regroupe les réseaux de soutien social et émotionnel que représentent la famille, les amis et d'autres institutions avec lesquelles la personne est liée. Selon les auteurs, ces dimensions peuvent avoir un rôle protecteur quand elles impliquent un soutien social, mais elles peuvent aussi présenter des risques si la personne endeuillée a de la difficulté à faire face aux circonstances de la mort ou si elle n’a pas suffisamment de soutien. 
Tableau 2. Type de décès, facteurs individuels et pratiques transnationales multilatérales

\begin{tabular}{|c|c|c|c|c|c|c|c|c|c|c|c|c|}
\hline & & & 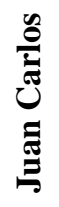 & 吾 & : & 总 & نَّ & 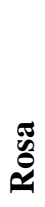 & $\stackrel{\Xi}{Z}$ & 䓪 & 莡 & 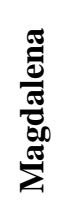 \\
\hline \multirow{3}{*}{\multicolumn{2}{|c|}{ Type de décès }} & Subit & $\checkmark \checkmark$ & $\checkmark$ & & $\bar{\nabla}$ & $\checkmark$ & $\checkmark$ & $\bar{\nabla}$ & $\checkmark \checkmark$ & $\checkmark$ & $\checkmark \checkmark$ \\
\hline & & Anticipé & & & $\checkmark$ & & & & & & & \\
\hline & & Violent & $\checkmark$ & $\checkmark$ & & $\checkmark$ & & $\checkmark$ & & & & $\checkmark$ \\
\hline \multirow{3}{*}{ 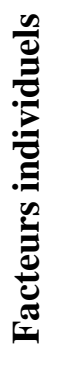 } & $\begin{array}{c}\text { de } \\
\text { protection }\end{array}$ & Religiosité & $\checkmark$ & $\checkmark$ & $\checkmark$ & $\checkmark$ & & & $\checkmark$ & $\checkmark$ & $\checkmark$ & \\
\hline & \multirow[b]{2}{*}{ de risque } & Migration forcée & $\checkmark$ & $\checkmark$ & & $\checkmark$ & $\checkmark$ & $\checkmark$ & $\checkmark$ & $\checkmark$ & $\checkmark$ & $\checkmark$ \\
\hline & & $\begin{array}{l}\text { Victime de mauvais } \\
\text { traitement de la part } \\
\text { des autorités }\end{array}$ & $\checkmark$ & $\checkmark$ & $\checkmark$ & $\checkmark$ & $\checkmark$ & $\checkmark$ & $\checkmark$ & $\checkmark$ & $\checkmark$ & $\checkmark$ \\
\hline \multirow{9}{*}{ 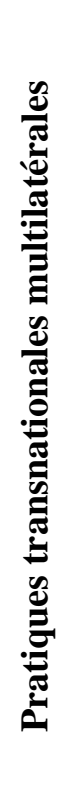 } & \multirow{4}{*}{$\begin{array}{c}\text { type } \\
\text { d'aide } \\
\text { apportée }\end{array}$} & Famille aux É.-U. & $\checkmark$ & $\checkmark$ & $\checkmark$ & & & $\checkmark$ & & & $\checkmark$ & $\checkmark$ \\
\hline & & $\begin{array}{l}\text { Famille dans le } \\
\text { pays d'origine }\end{array}$ & $\checkmark$ & $\checkmark$ & $\checkmark$ & $\checkmark$ & $\checkmark$ & $\checkmark$ & $\checkmark$ & & $\checkmark$ & $\checkmark$ \\
\hline & & $\begin{array}{l}\text { Compagnons de } \\
\text { voyage }\end{array}$ & & $\checkmark$ & & & & $\checkmark$ & $\checkmark$ & & $\checkmark$ & $\checkmark$ \\
\hline & & Refuge & $\checkmark$ & $\checkmark$ & & $\checkmark$ & & $\checkmark$ & $\checkmark$ & $\checkmark$ & $\checkmark$ & $\checkmark$ \\
\hline & \multirow{5}{*}{$\begin{array}{l}\text { réseaux } \\
\text { de } \\
\text { soutien }\end{array}$} & Économique & $\checkmark$ & $\checkmark$ & $\checkmark$ & & $\checkmark$ & $\checkmark$ & & & $\checkmark$ & \\
\hline & & Émotionnel & $\checkmark$ & $\checkmark$ & & $\checkmark$ & $\checkmark$ & $\checkmark$ & $\checkmark$ & & $\checkmark$ & $\checkmark$ \\
\hline & & $\begin{array}{l}\text { Psychologique ou } \\
\text { juridique }\end{array}$ & $\checkmark$ & $\checkmark$ & & $\checkmark$ & $\checkmark$ & & & $\checkmark$ & $\checkmark$ & $\checkmark$ \\
\hline & & Santé & & & & & & $\checkmark$ & & & & $\checkmark$ \\
\hline & & Religieux & & & & $\checkmark$ & $\checkmark$ & & $\checkmark$ & & & $\checkmark$ \\
\hline
\end{tabular}

Le tableau 2 montre les pratiques transnationales multilatérales existant dans les familles des participants. En effet, tous les participants ont des membres de leur famille ayant déjà émigré vers d'autres pays, bien que seulement six d'entre eux aient bénéficié de leur soutien actif. Par exemple, Rosa (femme, 34 ans) appartient à un véritable réseau transcontinental car, en plus 
du soutien matériel de ses sœurs installées aux États-Unis, sa mère reçoit au Honduras de l'argent d'un parent en Espagne, qu'elle lui renvoie ensuite au Mexique. Cette «intégration simultanée » que conceptualisent Glick Schiller et al. (1995 : 48) se retrouve également, bien que de manière plus traditionnelle, chez Juan Carlos (homme, 33 ans), Miguel (homme, 29 ans) et Damián (homme, 39 ans), qui reçoivent chacun une aide de parents à la fois dans leur pays d'origine et dans leur pays de destination. De son côté, Javier (homme, 24 ans) reçoit de l'argent de ses oncles et de ses cousins des États-Unis, mais il en renvoie une partie à sa famille au Salvador.

\section{Les circonstances du décès}

\section{Type de mort}

Le type de mort est significatif, car il détermine ce que sera l'expérience du deuil (Ayers et al. 2008; Rachédi et al. 2010). Les morts violentes et inattendues laissent d'importantes séquelles chez les migrants et les rendent vulnérables à la dépression (Folkman 1997; Fonnegra 2001; Rojas et Tuñón 2012; Yoffe 2013). Le tableau 3 rend compte des types de décès que les participants ont affrontés pendant leur processus migratoire.

Tableau 3. Expérience du deuil

\begin{tabular}{ll} 
Relation avec le défunt & \\
\hline Père/mère & 2 \\
Enfant & 1 \\
Grand-père/grand-mère & 4 \\
Cousin & 3 \\
Beau-père & 1 \\
Beau-frère & 1 \\
Type de décès & \\
\hline Assassinat & 7 \\
Maladie & 3 \\
Mort naturelle & 1 \\
Perdu dans le désert & 1
\end{tabular}


Un premier commentaire s’impose sur la relation entre le migrant endeuillé et le défunt. Les témoignages ne dénotent pas de différences dans l’impact émotionnel selon qu’il existe ou non un lien de sang avec le défunt (comme dans le cas d’un beau-père ou d’un beau-frère).

Cela s’explique par le fait que les familles d’Amérique centrale affrontent les difficultés du quotidien (crises et adaptations socioéconomiques permanentes) en développant des solidarités familiales et de fortes relations interpersonnelles qui vont plus loin que la famille nucléaire. La famille est conçue comme un groupe de personnes unies par une histoire sociale commune, elle-même traversée par des liens affectifs, qui se solidarisent dans leur relation aux institutions et à l'économie. En ce sens, la proximité affective est souvent puissante avec les membres qui n’ont pas de liens de sang, à partir du moment où ils partagent leur quotidien et s'intègrent et renforcent la structure d'entraide familiale, comme la belle-famille (d'ailleurs appelée de manière évocatrice « famille politique » en espagnol).

En contexte de migration, ces réseaux familiaux reposent souvent sur des ententes visant à aider celui qui émigre afin qu'il contribue par la suite au bien-être du reste de la famille, en mobilisant le capital social produit dans le lieu de destination et au lieu de provenance (Glick Schiller et al. 1992). Ces liens d'entraide matérielle ou émotionnelle se maintiennent malgré l'éloignement géographique et l'expérience du deuil peut même servir à les réactiver ou à les renforcer (Rachédi et al. 2016).

Par ailleurs, il convient de souligner que deux des participants ont connu des décès multiples dans leurs familles, ce qui complique le processus de deuil. Ainsi, le grand-père de Francisco (homme, 21 ans) est mort de manière « naturelle » (un arrêt cardiaque), mais à ce premier deuil 
s'est ajouté l'assassinat de son cousin lors d'une bagarre dans une fête. Dans des circonstances similaires, Rosa a connu deux morts violentes dans sa famille, l'une après l'autre. Tout d'abord son beau-frère a été assassiné deux semaines avant l'entretien, puis elle a perdu son cousin dans des circonstances traumatisantes : il a été exécuté devant son épouse et sa fille.

De plus, et bien que cela paraisse paradoxal, toutes les morts à la suite d'une maladie chronique ne sont pas forcément anticipées. Damián, par exemple, a vécu la mort de son père à la suite d'un cancer comme s'il s'agissait d'une mort soudaine, sa famille lui ayant caché sa maladie.

Ces témoignages concordent avec les résultats d'autres recherches antérieures qui associent une mort subite ou violente à un deuil complexe (Lundin 1984; Parkes 2001). Le migrant qui vit un deuil peut même voir sa santé physique et mentale affectée (Stroebe et al. 1993). Lorsqu'il vit des pertes inattendues, l'individu court le risque d'expérimenter une tristesse accrue et un sentiment de vide qui peuvent générer de l'anxiété et mener à la dépression. Aussi, l'absence lors des derniers moments du défunt peut engendrer un état émotionnel qui, à son tour, mène à ce qu’Achotegui appelle un « niveau de complication extrême du deuil » (2009 : 16). Il s’ensuit une difficulté à s’adapter à la vie sans la personne décédée, ce qui complique le deuil et peut même provoquer un sentiment de culpabilité (Barral 2002; Lundin 1984), comme dans le cas de Juan Carlos, qui se sent coupable de ne pas avoir trouvé la manière de convaincre son cousin du danger qui le menaçait :

Ils lui ont fait la même menace qu'ils m'ont faite à moi, [...] je lui ai dit que quand ces malosos [délinquants] menacent, c'est du sérieux, je lui ai dit «Allons-nous-en! » et il m'a répondu «Non, je vais rester un peu, je n'ai pas d'argent pour le moment.» «Allons-nous-en tout de même, on verra bien ce qu'on fera. » Et six jours plus tard, ils l'ont tué. [...] Je ressens beaucoup de tristesse parce que je n'ai pas réussi à le convaincre de me suivre. [...] Plus que tout parce que j'ai perdu un être cher que j'aimais trop... Je n'arrive toujours pas à m'en remettre. 
Rosa ressent la même culpabilité, dans son cas pour ne pas avoir pu éviter la mort de son beaufrère, assassiné parce qu’il l’a aidée à s’échapper du Honduras :

Ils ont assassiné celui qui m'a aidée à m'en aller. [...] Ils m'ont même envoyé une photo de lui, et je la regarde tout le temps, et cela me fait très mal. Je me sens comme si c'était arrivé hier et je me sens coupable de savoir que c'est de ma faute, que pour m'avoir aidée ils l'ont assassiné. Sa famille ne le sait pas, mais même cela me fait peur : que sa famille sache un jour que pour m'avoir aidée il a été assassiné et qu'ils [les membres de sa famille] veuillent me faire quelque chose.

De la même manière, un groupe de délinquants a mitraillé la grand-mère de Damián, en représailles contre lui :

Un gang l'a tuée [...]. Ils voulaient que je leur paye un "loyer » [extorsion] et ils m'avaient averti que sinon ils allaient tuer un membre de ma famille, mais jamais je n'aurais imaginé que ce serait ma grand-mère. [...] Ils l'ont tuée chez elle, ils sont entrés et lui ont tiré dessus.

Nous constatons que neuf des dix participants ont vécu un deuil traumatisant, qu'il ait été anticipé ou non. Seul Javier a rapporté la mort prévisible de son grand-père, malade depuis longtemps :

Quand je suis parti, sa santé était déjà très détériorée et les médecins ne lui donnaient plus d'espoir. [...] Il y a un moment où il faut prendre des décisions et si on les prend pour une raison spéciale, je pense qu'à la fin, le sacrifice aura sa récompense. C'est ce que j'ai pensé au moment de sa mort, et c'est pour ça que je suis encore ici. [...] C’était le mieux pour lui parce qu'il avait beaucoup souffert de cette maladie qui le détruisait de son vivant.

De manière attendue, l'adaptation et la continuation du projet migratoire ont été plus faciles pour lui que pour les autres participants, puisque dans le cas d'une maladie le processus de deuil commence dès le diagnostic (Yoffe 2013). 


\section{$\underline{\text { Les stratégies d'adaptation }}$}

Quel mécanisme et quelles ressources les migrants utilisent-ils pour faire face à leur deuil? En nous basant sur les informations recueillies, nous observons que chaque participant tend à construire sa propre stratégie d'adaptation à la perte selon l'évaluation qu'il effectue de la situation et des ressources qu'il a à sa portée. Ces stratégies vont de l'isolement social (essayer de s’éloigner du problème) à l'autocontrôle (tenter de contrôler ses propres réponses émotionnelles), en passant par la fuite (abus d'alcool ou de drogues) ou la réévaluation positive des événements (se convaincre que tout ira bien).

Nous avons regroupé ces stratégies en deux catégories : celles qui engagent un changement de comportement et celles qui impliquent un processus de régulation émotionnelle.

La première catégorie implique une modification des comportements. Plusieurs migrants ont mentionné avoir essayé de substituer leurs pensées douloureuses par des activités, nouvelles ou non, mais pas forcément saines pour eux : elles servent seulement à éluder l'événement tragique. Ainsi, les participants ont mentionné que leur consommation de drogues légales et illégales, en particulier d’alcool et de marijuana, a augmenté considérablement.

Dans un autre type de stratégie comportementale, Rosa raconte avoir ajusté ses activités quotidiennes pour s’immerger totalement dans le travail et ne pas penser à ce qui était arrivé, essayant ainsi d'éluder son sentiment de culpabilité : «Mon travail m’a beaucoup aidée, me maintenir occupée m'aide à ne pas trop penser. [...] C’est très important de me maintenir occupée, parce que si je m'enferme, je me sens seule et je pense à la réalité de la situation. Ça m’affecte plus. » 
Miguel, de son côté, exprime un sentiment d’impuissance qui l’a amené à s’isoler : « Je n'arrive pas à trouver une sortie et des forces. [...] Pour m'en sortir, je veux être seul et ne voir personne. [...] Je ne me suis approché de personne. » Pour certains migrants comme Miguel, l'isolement sert de protection contre la souffrance et l'incompréhension. Par ailleurs, la plupart d'entre eux sont déjà habitués à la solitude, car elle les protège des risques de la migration irrégulière, dans laquelle le migrant est doublement stigmatisé : d'une part, il est jugé comme délinquant alors qu'il fuit justement la violence, et d'autre part, on exige constamment qu’il justifie sa décision d’émigrer sans documents valides.

Sur le plan symbolique, l'émotion liée à la perte d'un être cher réactive aussi le sens des origines et de l'attachement à la famille. Ainsi, Magdalena (femme, 38 ans) rapporte qu'après la mort de son fils, elle parlait tous les jours au téléphone avec sa mère restée au Honduras, malgré les difficultés et le coût de la communication. Dans ce cas, le deuil a fait office d'élément fédérateur et régénérateur pour le groupe social dans son ensemble et a permis de renforcer les relations avec la parentèle restée au pays.

Le second type de stratégie se fonde sur une tentative de régulation de la profonde tristesse que tous les participants ont exprimée, comme Gabriel (homme, 25 ans), qui met l'accent sur « la préoccupation, le stress, la dépression. [...] [La préoccupation] pour les enfants, pour nous, pour mes neveux » et la nostalgie du défunt, comme sa mère qui lui envoyait chaque jour « dix, quinze messages, des messages audio, qui me demandaient pourquoi je n'avais pas donné de nouvelles ».

Nous avons observé un mécanisme de lutte contre l'angoisse : la conviction que tout va bien se passer et la négation du besoin d'aide, même lorsque celui-ci est évident. Alors qu’un 
psychologue lui a recommandé et proposé de suivre une thérapie pour pouvoir supporter la double perte soudaine de son grand-père et de son cousin, Francisco a refusé : « J'ai été face à la mort, je n'en ai pas peur. D’ailleurs, j’y suis préparé. À n'importe quel jour, lieu et moment. Je ne m'en fais pas pour la mort, sincèrement. [...] Je n'ai pas besoin de soutien émotionnel ni de psychologue, je vais bien. »

Cette négation du besoin d'aide va de pair avec un certain fatalisme menant à accepter la perte telle qu'elle est arrivée. C’est ainsi que Magdalena affirme : «C’est le destin de la vie. Tout est écrit d'avance. Mon sort est de me résigner, d'accepter, même si c'est dur. »

Les facteurs individuels de protection et de risque

\section{Une forte religiosité...}

En plus des stratégies d'adaptation au deuil, certains facteurs individuels peuvent avoir un effet protecteur ou, au contraire, un effet générateur de risque pour la personne qui les expérimente. Dans notre étude, les croyances religieuses et l'appartenance à un groupe religieux se sont démarquées comme facteurs de protection. En effet, tous les participants, sauf un, ont déclaré pratiquer une religion, et sept d'entre eux ont montré des marqueurs prononcés de croyances religieuses, dont ils ont fait leur principale source d'acceptation de la perte d'un proche, comme l’illustre le témoignage de Javier : «Si Dieu me donne un poids à porter, c'est parce que je peux le supporter, et si j'en suis là, c'est parce que je vais m'en sortir. Je fais des efforts avec l'âme un peu frappée, mais je continuerai. »

Les croyances religieuses servent de « soupape thérapeutique » pour les migrants endeuillés et leur permettent d'exprimer par la prière la douleur qu'ils ont souvent du mal à verbaliser : « Je 
me déclare catholique à $100 \%$. [...] J'implore constamment Dieu et j'ai totalement confiance en lui. Je me sens bien quand je prie. Je crois en Dieu. » (Ana, femme, 31 ans)

Même Miguel, qui affirme ne pratiquer aucune religion, dit craindre Dieu : «Je ne pratique aucune religion [...], mais je crains Dieu; je sais que Dieu existe et que c'est lui qui nous donne la vie, que c'est lui qui m’a maintenu et protégé jusqu'à ce moment précis de ma vie. » Ce témoignage nous rappelle que même si nous observons que les pressions de la globalisation dans les sociétés modernes tendent à effacer les caractéristiques religieuses et culturelles, le deuil en contexte de migration renforce les valeurs et les croyances traditionnelles.

Le cas de Rigoberto (homme, 18 ans) attire l'attention, car cet homme voue un culte à la Santa Muerte, qui lui donne confiance et force dans l'adversité : «Pour moi, la mort est ce qu'il y a de plus saint [...] parce qu'elle me donne tout. Pour les autres non, parce que c'est la mort et ils ont peur d'elle, par contre moi non. La mort me donne tout ce que je lui demande, parce que moi, je fais tout ce qu'elle me demande. »

Nous constatons donc que pour affronter la douleur de l'éloignement de la famille et des amis, la religiosité occupe un rôle fondamental (même quand il s’agit de croyances populaires, comme dans le cas de Rigoberto). Très présente dans les sociétés hondurienne et salvadorienne (dont les habitants se déclarent religieux à $93 \%$ et $83 \%$ respectivement), la religion sert de catalyseur de résilience personnelle aux migrants, leur fournissant des outils de réconfort et de résignation. Les pratiques socioreligieuses permettent alors de faire face à la souffrance et donnent un sens symbolique à la séparation, de même qu’elles assurent la cohésion sociale du groupe en contexte de migration (Kokou-Kpolou et al. 2017). 


\section{... qui se traduit dans des rituels transnationaux}

Pour les migrants, malgré leur forte religiosité, il est difficile de maintenir leurs pratiques religieuses dans le contexte dynamique de la migration, en raison d'un manque de temps et de l'éloignement de leur lieu de culte habituel. Comme les contacts avec la communauté religieuse d'origine sont sporadiques ou inexistants, la religion constitue une ressource difficile à mobiliser. Or, une partie importante du deuil est l'acceptation de la mort, qui se vit au moyen des rites funéraires qui permettent de faire les adieux au défunt (Chéron-Leboeuf et al. 2016). Face à l’impossibilité d’assister aux funérailles (en raison de la distance, du coût, du risque ou du renoncement au projet migratoire que cela impliquerait), certains migrants adaptent leurs pratiques religieuses à leur situation du moment.

Nous avons mis en évidence des pratiques rituelles transnationales « multisites » et simultanées entre la cérémonie dans le pays d’origine et la cérémonie du lieu de transit, donnant ainsi des « bricolages rituels » (Petit 2002, citée par Rachédi et al. 2010), comme ce fut le cas après la mort du fils de Magdalena. En effet, au moment même où avaient lieu ses funérailles au Honduras, une messe était célébrée dans la paroisse mexicaine à laquelle appartenait le refuge où Magdalena se trouvait à ce moment-là. « Cela m’a aidée à trouver la paix », explique-t-elle. On voit bien ici que les rituels constituent des mécanismes de défense et que leur fonction symbolique est de « donner un sens » à l’absence du défunt (Kokou-Kpolou et al. 2017).

Par ailleurs, Gabriel et Francisco ont réussi à déjouer la distance en utilisant la technologie, qui leur a permis de conserver un lien actif avec des acteurs stratégiques de leur communauté religieuse au Honduras. De cette façon, Gabriel maintient un contact avec un «groupe de développement » de son église d’origine, ses membres l'ayant toujours soutenu : 
[J'appartiens à] un groupe de développement. Nous passons beaucoup de temps ensemble, physiquement là-bas et maintenant ici. [...] Ils se réunissent pour tout. Si par exemple c'est ton anniversaire, ils te font une fête, te rendent visite. [...] Ils prennent de mes nouvelles, font attention à moi. Ils se rendent compte de tout ce dont j'ai besoin.

Le témoignage de Francisco va dans le même sens. Il maintient une communication régulière avec un prêtre de l’armée hondurienne :

Dans le bataillon, quand j'y étais, il y avait un prêtre. Il nous fournissait des enseignements sur l'église et ce genre de choses. J'avais une grande confiance en lui et c'est avec lui que je parle par internet. Il me donne beaucoup de conseils, me dit ce que je dois faire et pas faire, que je dois réfléchir avant d'agir. [...] [Je parle] très souvent avec lui.

Francisco le dit explicitement : s’il continue à parler avec son prêtre du Honduras, c'est parce qu'il a « une grande confiance en lui ». Les prêtres ne manquent pourtant pas sur son chemin au Mexique, en particulier parce que la quasi-totalité des refuges par lesquels il est passé sont liés à une église qui offre un soutien spirituel, mais ils ne suscitent pas ce sentiment de confiance qui caractérise son prêtre du Honduras. De ce fait, nous constatons que la confiance détermine la qualité et l'utilité des réseaux de soutien social; ce n’est pas nécessairement la taille ou l'extension du réseau qui compte, mais la perception qu’en a le migrant.

\section{Les facteurs interpersonnels de protection}

La présence d’organisations de la société civile est fondamentale pour que les migrants trouvent les moyens de vivre leur deuil sans renoncer à leur projet migratoire. Il existe de nombreuses études sur l'effet positif des réseaux sociaux dans la construction de la résilience (Armstrong et al. 2005; Black et Lobo 2008; Walsh 2006). De la même manière, l’insuffisance de soutien social explique pourquoi certaines personnes ne parviennent pas à dépasser un événement traumatisant (Brewin et al.2000; Echeburúa et al. 2011; Ozer et al. 2003; Yoffe 2013). 


\section{Les effets du décès sur les réseaux familiaux}

Les personnes migrantes appartiennent à « un ensemble de réseaux entrelacés » (Levitt et Glick Schiller 2004 : 1006) dont la famille constitue la base. Dans le contexte du transit, elles ne bénéficient que de très peu de soutien émotionnel et matériel, particulièrement celles qui sont en situation irrégulière et qui n’ont accès à aucun service social. Le soutien émotionnel de la famille est alors déterminant, car il donne aux migrants le sentiment d’être aimés, protégés et valorisés (Worden 2004). Voilà pourquoi, en cas de deuil, leurs solidarités familiales et leurs réseaux d'entraide se raffermissent de manière bien plus vigoureuse que lors de deuils de nonmigrants.

La famille peut même constituer l'unique source de soutien pour des migrants isolés, comme dans le cas de Damián, qui explique n’avoir «aucun ami, juste [s] a famille ». Dans des cas extrêmes, le soutien familial se résume à une seule personne, comme le relate Rigoberto : « Ma mère, c'est tout ce que j'ai dans la vie [...], et c'est dur quand tu es loin d'elle, sans savoir de quoi demain sera fait. »

On voit ici que le deuil entraîne parfois des tensions, voire des fragmentations dans toute la structure familiale, ce qui peut modifier la trajectoire migratoire (Fernández et al. 2016). Ce fut le cas de Francisco, de Miguel et de Javier, qui ont dû réajuster leurs projets pour aider leurs familles en cherchant de nouvelles sources de revenus. Ce changement est encore plus radical quand le défunt occupait une fonction centrale dans le réseau transnational (Ariza 2014), comme dans le cas de Rigoberto, qui a dû interrompre son projet d’aller vers les États-Unis pour travailler au Mexique et substituer l’unique source de revenus de sa mère. L'expérience du deuil est collective et elle peut transformer les liens familiaux et les relations entre chaque 
membre. La disparition du défunt redéfinit donc les stratégies familiales collectives et oblige les membres du réseau familial à réviser leurs fonctions respectives pour couvrir les besoins de la famille d'une nouvelle façon.

Ces témoignages montrent l'impact qu'a la mort d'un parent proche sur l'ensemble de la famille, celle-ci fonctionnant comme un réseau transnational interdépendant dans lequel la disparition d'un élément affecte l'ensemble du réseau, tant les personnes qui migrent que celles qui ne migrent pas (De Haas 2010; Diminescu 2002; Stark et Bloom 1985).

\section{$\underline{\text { Les refuges, piliers des réseaux de soutien de la société civile }}$}

Dans les témoignages recueillis, les refuges apparaissent comme des institutions productrices de capital social. En effet, les dix personnes interrogées ont mentionné spontanément que ce sont les institutions en lesquelles ils ont le plus confiance. Comme nous l'avons vu avec Ana, en plus de l'assistance humanitaire qu'ils fournissent (une auberge pour se restaurer et se reposer en sécurité), les refuges dynamisent les liens sociaux des migrants, car ils représentent un capital social que ces derniers ne pourraient pas constituer eux-mêmes et qui leur est utile au moment d’affronter les conséquences économiques (comme le coût de l’organisation d’une cérémonie religieuse) et psychologiques associées à la mort d'un parent. C’est particulièrement vrai quand le migrant endeuillé choisit l'isolement comme stratégie de protection contre l’angoisse causée par l'absence de l'être cher (Kokou-Kpolou et al. 2017).

Ainsi, les refuges constituent des relais sociaux, familiaux, religieux et juridiques qui servent de contrepoids à la clandestinité et compensent les responsabilités que l’État ignore ou ne peut prendre en charge (par manque de ressources, de capacités ou de volonté). De la même manière, ils proposent des services que l'État offre bel et bien aux personnes en situation irrégulière 
(comme le service médical par l’intermédiaire du Seguro Popular ${ }^{5}$ ), mais que les migrants ne souhaitent pas utiliser par crainte d'une dénonciation ou d'une détention.

Là encore, le facteur « confiance » est fondamental. On le retrouve dans le témoignage d’Ana, qui a modifié ses projets initiaux à la suite d'une promesse faite par un prêtre rencontré dans le refuge de Ciudad Ixtepec : «Nous avions tout d'abord un lieu où aller à Colima, mais un prêtre nous aide et il va nous fournir un emploi à Mexico. » Les refuges, en grande majorité dirigés par des religieux, donnent accès à de nombreuses ressources qui dépassent le soutien spirituel. Ainsi, Ana est tout d'abord entrée en contact avec ce prêtre pour trouver un soutien purement religieux après la mort de son beau-père. Paradoxalement, cet événement tragique lui aura permis, par l'entremise du prêtre, de renforcer sa capacité «à mobiliser en [sa] faveur le capital d'un groupe plus ou moins institutionnalisé et riche en capital » (Bourdieu 2000 : 149, notre traduction).

En complément à sa dimension spirituelle, l'aide fournie par ces institutions religieuses a une dimension sociale. Les femmes et les hommes d’Église représentent un soutien déterminant pour ceux qui, en raison de leur situation irrégulière, n’ont pas accès à d'autres types de services. Le prêtre cristallise la confiance et devient un personnage stratégique, un point d’interconnexion avec d'autres instances qui permet de satisfaire les nécessités fondamentales des migrants : rencontrer un avocat qui les guidera dans leurs démarches (cinq participants ont bénéficié d’un appui juridique par cet intermédiaire); voir un médecin ou un psychologue qui les accompagnera dans le processus d'adaptation à la perte d'un proche, ou trouver un emploi qui leur permettra de subvenir à leurs besoins et de couvrir éventuellement les frais occasionnés par le décès. 
L'histoire de Magdalena illustre parfaitement le rôle compensateur du refuge qui l'a aidée à faire face à son manque d’intégration sociale, plus encore que sa propre famille : « [La coordinatrice du refuge] m'a aidée avec tout. [...] Aux États-Unis, nous n'avons plus personne qui nous aide. Nous avons bien de la famille mais ils ne nous aident plus, parce que, soi-disant, ils n'ont plus d'argent. »

Lorsque son fils est mort dans le désert de Sonora après s’être perdu alors qu'il essayait de faire passer un sac plein de drogue du côté des États-Unis, le refuge a aidé Magdalena avec le rapatriement du corps au Honduras. Durant cet événement, la coordinatrice du refuge a fait office de lien entre les différentes instances nationales et internationales : le consulat du Honduras à Dallas pour obtenir l'acte de décès, la Comisión Nacional de Derechos Humanos pour l'aider à obtenir un visa temporaire, et la compagnie funéraire du Honduras qui avait oublié d'apposer son tampon sur le certificat de sépulture. Après cela, lorsque la fille de Magdalena est née au Mexique, la coordinatrice l’a également orientée vers le bureau d'état civil.

Loin de nous l'idée de dépeindre cette migrante comme passive et dépendante des actions d'une autre personne détentrice de l’information : au contraire, Magdalena nous a révélé que, grâce à ces contacts, elle peut maintenant suivre elle-même, et de près, ses propres procédures en cours, montrant de la sorte des signes d'émancipation :

Je me suis mise en contact avec la consule du Honduras à Dallas, elle m'a dit qu'elle me tiendrait au courant. [...] Ici, ils [le personnel du refuge] m'ont aidée à entrer en contact avec les services de santé. [...] J'y suis allée seule, ils m'ont prise en charge et le docteur m'a envoyé au Seguro Popular pour m'y inscrire. Je suis alors allée me faire enregistrer à l'hôpital où j'ai accouché. [La coordinatrice] m'a aidée avec l'état civil de ma fille. [...] Elle m'a emmenée à l'édifice de l'état civil. [...] Grâce à elle, je suis allée aux Droits de l'Homme [bureau de la Comisión Nacional de Derechos Humanos], où j'ai pu m'entretenir avec une responsable. 
Le refuge a donc joué un rôle de transmetteur et de canalisateur d’informations pour Magdalena, lui permettant ainsi d'améliorer sa situation par la création de capital social. Il s’agit donc d’un réseau crucial, qui se combine avec les réseaux transnationaux (notamment la famille), car il oriente et conseille les migrants et leur fournit de l'aide en cas d'urgence (Chéron-Leboeuf et al. 2016).

\section{Conclusions}

Sur le deuil

Notre travail présente une perspective exploratoire des conditions d'accompagnement des migrants endeuillés au Mexique, au moyen d'une analyse descriptive des principaux facteurs de risque et de protection associés au deuil, ainsi qu’une discussion sur le rôle des réseaux de soutien social durant ce processus. Cette recherche apporte une contribution à un nouveau champ de réflexion sur l'expérience du deuil chez les migrants en transit, population sur laquelle il est nécessaire de mener plus de recherches afin d'en comprendre les besoins spécifiques.

En effet, nous avons vu que tout immigrant endeuillé souffre d'un « cumul de deuils » (Rachédi et al. 2016). La souffrance des personnes en situation irrégulière et en transit est plus difficile à reconnaître que celle des immigrants réguliers ou arrivés à destination, car celles-ci souffrent en plus de la crainte permanente d’être déportées, ce qui les oblige à vivre dans la clandestinité.

De manière concordante avec les recherches antérieures, nous constatons qu'il n’y a pas un facteur déterminant pour les migrants qui ont à s’adapter à la perte d'un être cher, mais plutôt une somme de facteurs de protection et de risque (Sameroff et al. 2003). Dans le cas spécifique des migrants clandestins, les facteurs de risque abondent (en raison de leur forte 
marginalisation sociale) et ils sont plus nombreux que les facteurs de protection qui déterminent la capacité individuelle de résilience.

Évaluer ces facteurs en profondeur requiert donc d'étudier l'histoire, la trajectoire et les réseaux des migrants transnationaux au travers desquels ils construisent de nouveaux champs sociaux et un «mode de vie transnational » (Guarnizo 2003 : 669). En ce sens, il ne faut pas concevoir le processus de deuil comme un absolu, résolu ou non, assimilé ou encore cause de souffrance : les deuils des migrants sont partiels et de natures variées. Comme l'explique Bonanno (2004 : 20, « there are multiple and sometimes unexpected pathways to resilience ».

Sur les réseaux de soutien social

Ainsi, penser le deuil en contexte de mobilité implique de ne pas seulement se concentrer sur le comportement de l'individu migrant, mais aussi sur les liens qu'il maintient avec sa famille, avec ses amis et avec ses communautés d'appartenance. Nous avons mis en lumière de véritables stratégies familiales transnationales, indispensables à cause de l'absence partielle des États, qui n’interviennent que dans les aspects matériels de la mort (le rapatriement du corps, par exemple), mais qui n’accompagnent pas les survivants. Les réseaux de soutien social transnationaux renforcent donc la capacité d'adaptation des migrants et compensent en partie la « relation directe et sans équivoque » que présente Achotegui (2004 : 39) entre la migration et l'ensemble des deuils symboliques.

Les refuges sont souvent le principal soutien des participants. Ils offrent un espace social où ces derniers peuvent exprimer librement leur douleur, et ils aident à réduire les coûts sociaux, psychologiques et économiques de la migration irrégulière. Ils jouent un rôle fondamental dans la gestion du stress et de l'angoisse du deuil, ainsi que dans l'accompagnement socioreligieux, 
et ils ont un impact positif sur la qualité de vie des sujets durant leurs trajectoires migratoires. À la différence du capital culturel ou économique, le capital social qu’offrent les refuges est accessible pour les personnes les plus vulnérables.

Enfin, les réseaux sociaux sont des facteurs qui peuvent moduler l'impact du décès de manière favorable, mais aussi de façon défavorable. L'histoire du fils de Magdalena, mort après s’être rapproché d’un groupe de trafiquants de drogue pour aider sa mère, confirme ce que Krissman (2005) avance : un groupe délinquant constitue aussi un réseau social, ce qui montre bien que tous les réseaux n’ont pas forcément un effet positif.

La construction de capital social, une solution?

Nous avons constaté que les participants qui se sont le mieux adaptés à la perte d'un parent proche sont tous passés par la construction d'un capital social important. Au moment d'établir des contacts stables et de confiance avec des réseaux de soutien, ces migrants ont commencé à reprendre le contrôle de leur vie et à assimiler la réalité de la perte.

Notre étude a mis l'accent sur l'importance de la confiance dans cette construction. La confiance est un facteur déterminant au moment d'utiliser les relations comme ressource, puisqu'elle « facilite la coordination et la coopération » (Putnam 1995 : 172). Il s’agit là d’une tâche difficile toutefois, car, comme l'expliquent Sacipa et al. (2007), la migration forcée renforce la tristesse et la méfiance des migrants. 


\section{Bibliographie}

Achotegui, J., 2009. « Migración y salud mental. El síndrome del inmigrante con estrés crónico y múltiple (síndrome de Ulises) », Zerbitzuan, n 46, p. 163-169.

Achotegui, J., 2004. « Migrar en condiciones extremas: El síndrome de Ulises », Revista Norte de salud mental, $\mathrm{n}^{\circ} 21, \mathrm{p} .39-52$.

Ariza, M., 2014. "Care Circulation, Absence and Affect in Transnational Families », in L. Baldassar et L. Merla (dir.), Transnational Families, Migration and the Circulation of Care. Understanding Mobility and Absence in Family Life. New York, Routledge, p. 94-115.

Armstrong, M. I., S. Birnie-Lefcovitch et M. T. Ungar, 2005. "Pathways between Social Support, Family Well-Being, Quality of Parenting, and Child Resilience: What we Know », Journal of Child and Family Studies, vol. 14, n² 2, p. 269-281.

Ayers, T., I. Sandler et S. Wolchik, 2008. «Resilience rather than Recovery: A Contextual Framework on Adaptation Following Bereavement », Death Studies, vol. 32, n 1, p. 59-73.

Bar de Jones, G., 2001. « La migración como quiebre vital ». Communication présentée au II Congreso Argentino de Psicoanálisis de familia y pareja, Buenos Aires, Argentina. http://www.bazenbehin.net/kulturartean/pictures/cajaherramientas/la_migracion.pdf [consulté le 28 décembre 2016].

Barral, $\quad$ M., $2002 . \quad$ La Psicología del Migrante ». http://www.vicariadepastoral.org.mx/social/comision_migrantes/anexos/PSICOLOGIA_del_ Migrante.pdf [consulté le 14 février 2017].

Bennegadi, R., 1984. " Alcoolisation des travailleurs migrants en Europe », Migrations Santé, vol. 38, p. 19-22.

Bertrand, D., 1990. " Projet d'exil et acquisition de la langue étrangère », Cahiers du CERESI, n 4, p. 139-147.

Bhugra, D. et K. Bhui (dir.), 2018. Textbook of Cultural Psychiatry. Cambridge, Cambridge University Press.

Black, K. et M. Lobo, 2008. « A Conceptual Review of Family Resilience Factors », Journal of Family Nursing, vol. 14, n 1, p. 33-55.

Bonanno, G., 2004. «Loss, Trauma and Human Resilience. Have we Underestimated the Human Capacity to Thrive after Extremely Aversive Events? ", American Psychologist, vol. $59, \mathrm{n}^{\circ} 1$, p. 20-28.

Bourdieu, P., 2000. «Las formas del capital. Capital económico, capital cultural y capital social », in P. Bourdieu, Poder, derecho y clases sociales. Barcelona, Desclée, p. 148-149.

Brewin, C. R., B. Andrews et J. D. Valentine, 2000. « Meta-Analysis of Risk Factors for Posttraumatic Stress Disorder in Trauma-Exposed Adults ", Journal of Consulting and Clinical Psychology, vol. 68, n 5, p. 748-766. 
Cabodevilla, I., 2007. « Las pérdidas y sus duelos », Anales del Sistema Sanitario de Navarra, vol. 30, n 3 , p. 163-176.

Casado, B. L. et P. Leung, 2001. « Migratory Grief and Depression among Elderly Chinese American Immigrants », Journal of Gerontological Social Work, vol. 36, n 1-2, p. 5-26.

Chéron-Leboeuf, L. et al., 2016. Mort et deuil en contexte migratoire : guide d'informations. SHERPA. http://www.sherpa-recherche.com/wp-content/uploads/2016/11/GUIDE-Mortdeuil.pdf [consulté le 19 octobre 2018].

Comisión Nacional de Derechos Humanos, 2011. Secuestros de personas migrantes centroamericanas en tránsito por México. http://www.cndh.org.mx/sites/all/doc/Informes/Especiales/2011_secmigrantes.pdf [consulté le 17 avril 2017].

De Haas, H., 2010. « Migration and Development: A Theoretical Perspective », International Migration Review, vol. 44, $\mathrm{n}^{\circ}$ 1, p. 227-264.

De la Revilla, L. et al., 2011. «Estudio del duelo migratorio en pacientes inmigrantes que acuden a las consultas de atención primaria. Presentación de un cuestionario de valoración del duelo migratorio, Atención Primaria », Atención Primaria, vol. 43, n 9, p. 467-473.

Diminescu, D., 2002. "Les migrations à l'âge des nouvelles technologies », Hommes \& Migration, $\mathrm{n}^{\circ} 1240$, p. 6-9.

Echeburúa, E., P. de Corral et P. J. Amor, 2005. « La resistencia humana ante los traumas y el duelo ", Colegio Oficial de Trabajo Social de Badajoz, p. 339-359. https://www.researchgate.net/publication/265248210_LA_RESISTENCIA_HUMANA_ANT E_LOS_TRAUMAS_Y_EL_DUELO [consulté le 24 janvier 2019].

Estrada, A., 2012. Una aproximación a las reacciones psicológicas en la migración. Facultad Latinoamericana de Ciencias Sociales. http://www.flacso.org/secretaria-general/unaaproximaci-n-reacciones-psicol-gicas-migraci-n [consulté le 10 avril 2017].

Falicov, C., 1999. « Clínica de las familias migratorias ». Entretien realisé par Ricardo Ramos Gutiérrez, Perspectivas Sistémicas. La Nueva Comunicación, nº 64, p. 3-4.

Fernández, M., M. Orozco et D. Heras, 2016. «Familia y migración : las familias transnacionales », Familia. Revista de ciencias y orientación familiar, n 53, p. 87-106.

Fernández de Castro, R., 2012. " Transmigración y violencia en México », Harvard Review of Latinamerica. https://revista.drclas.harvard.edu/book/transmigraci\%C3\%B3n-y-violencia-en-

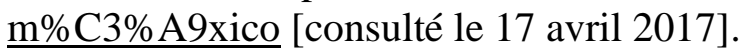

Folkman, S., 1997. «Positive Psychological States and Coping with Severe Stress », Social Science and Medicine, vol. 45, n ${ }^{\circ}$, p. 1207-1221.

Fonnegra, I., 2001. De cara a la muerte. Editorial Andres Bello. 
Glick Schiller, N., L. Basch et C. Blanc-Szanton (dir.), 1992. Towards a Transnational Perspective on Migration. Race, Class, Ethnicity and Nationalism Reconsidered. New York, Annals of the New York Academy of Sciences.

Glick Schiller, N., L. Basch et C. Blanc-Szanton, 1995. «From Immigrant to Transmigrant: Theorizing Transnational Migration », Anthropological Quarterly, vol. 68, n 1, p. 48-63.

Grinberg, L. et R. Grinberg, 1986. Psychanalyse du migrant et de l'exilé. Lyon Césura Lyon Éditions.

Guarnizo, L. E., 2003. "The Economics of Transnational Living », The International Migration Review, vol. 37, n 3, p. 666-699.

Infante, C. et al., 2012. «Violence Committed against Migrants in Transit: Experiences on the Northern Mexican Border », Journal of Immigrant and Minority Health, vol. 14, n 3, p. 449459.

Insight Crime, 2016. Balance de Insight Crime Sobre Homicidios en Latinoamérica en 2016, Unidad Investigativa de Venezuela. http://es.insightcrime.org/analisis/balance-insight-crimesobre-homicidios-2016 [consulté le 30 octobre 2017].

Jimenez, V., 2016. «La violencia en el Triángulo Norte de Centroamérica: una realidad que genera desplazamiento », Papel Político, vol. 21, n 1 p. 167-196.

Kogan, I., 2010. « Migration and Identity: Different Perspectives », International Journal of Psychoanalysis, vol. 91, n 5, p. 1206-1208.

Kokou-Kpolou, K. et al., 2017. «A Cross-Cultural Approach to Complicated Grief Reactions Among Togo-Western African Immigrants in Europe ", Journal of Cross-Cultural Psychology, vol. 48, n 8, p. 1247-1262.

Krissman, F., 2005. "Sin Coyote ni Patrón: Why the "Migrant Network" Fails to Explain International Migration », The International Migration Review, vol. 39, n 1, p. 4-44.

Kuhner, G., 2011. «La violencia contra las mujeres migrantes en tránsito por México », Dfensor. Revista de derechos humanos, $\mathrm{n}^{\circ} 6, \quad$ p. 19-25. http://www.corteidh.or.cr/tablas/r26820.pdf [consulté le 9 mai 2017].

Levitt, P. et N. Glick Schiller, 2004. «Conceptualizing Simultaneity: A Transnational Social Field Perspective on Society », International Migration Review, vol. 38, n 3, p. 1002-1039.

Lundin, T., 1984. «Morbidity Following Sudden and Unexpected Bereavement », British Journal of Psychiatry, $\mathrm{n}^{\circ}$ 144, p. 84-88.

Médicos Sin Fronteras, 2014. Casi el 60 \% de los migrantes centroamericanos atendidos en México ha sufrido episodios de violencia. http://www.msf.mx/web/MSFcasi_el_60_de_los_migrantes_centroamericanos_atendidos_en_mexico_ha_sufrido_episodio s_de_violencia [consulté le 22 avril 2017]. 
Mejía, S. et L. Cruz, 2014. "Violencia institucional contra los transmigrantes centroamericanos », Revista trabajo social UNAM, n 6, p. 45-65.

Michel, L., 2015. « Migration et addictions : aspects cliniques ", L'information psychiatrique, vol. $91, \mathrm{n}^{\circ} 1$, p. $21-28$.

Organisation internationale pour les migrations, 2010. Documento de trabajo: migración y transnacionalismo.

http://www.iom.int/jahia/webdav/shared/shared/mainsite/microsites/IDM/workshops/migrati on_and_transnationalism_030910/background_paper_es.pdf [consulté le 2 avril 2017].

Ozer, E. J. et al., 2003. « Predictors of Posttraumatic Stress Disorder and Symptoms in Adults: A Meta-Analysis », Psychological Bulletin, vol. 129, n 1, p. 52-73.

Parkes, C., 2001. " A Historical Overview of the Scientific Study of Bereavement », in M.S. Stroebe et al. (dir.), Handbook of Bereavement Research: Consequences, Coping, and Care. Washington, American Psychological Association, p. 25-45.

Petit, A., 2002. « L’ultime retour des gens du fleuve Sénégal », Hommes \& Migration, n 1236, p. 44-52.

Putnam, R., 1995. «Bowling alone: America's Declining Social Capital », Journal of Democracy, $\mathrm{n}^{\circ}$ 6, p. 65-78.

Rachédi, L., C. Montgomery et B. Halsouet, 2016. « Mort et deuil en contexte migratoire : spécificités, réseaux et entraide ", Enfances Familles Générations, $\mathrm{n}^{\circ} 24$. https://journals.openedition.org/efg/1041\#text [consulté le 2 novembre 2018].

Rachédi, L., J. Le Gall et V. Leduc, 2010. « Réseaux transnationaux, familles immigrantes et deuils », Lien social et Politiques, n 64, p. 175-187.

Reyes, C., 2011. « Historia y actualidad del culto a la Santa Muerte », El Cotidiano, n 169, p. 51-57.

Rojas, M. et P. Tuñón (dir.), 2012. Género y Migración I. San Cristóbal de Las Casas, El Colegio de la Frontera Sur, El Colegio de la Frontera Norte, A. C., El Colegio de Michoacán, A.C., Centro de Investigaciones y Estudios Superiores en Antropología Social.

Sacipa, S. et al., 2007. «Psychological Accompaniment to Liberate the Suffering Associated with the Experience of Forced Displacement », Universitas Psychologica, vol. 6, n 3, p. 589600 .

Sameroff, A. J., L. M. Gutman et S. C. Peck, 2003. « Adaptation among Youth Facing Multiple Risks: Prospective Research Findings ", in S. Luthar (dir.), Resilience and Vulnerability: Adaptation in the Context of Childhood. New York, Cambridge University Press, p. 364-391.

Stark, O. et D. Bloom, 1985. "The New Economics of Labor Migration », The American Economic Review, vol. 75, n² 2, p. 173-178. 
Stroebe, M. S. et H. Schut, 2001. « Models of Coping with Bereavement: A Review », in M. S. Stroebe et al. (dir.), Handbook of Bereavement Research: Consequences, Coping and Care. Washington, American Psychological Association, p. 375-404.

Stroebe, M. S., W. Stroebe et R. O. Hansson (dir.), 1993. Handbook of Bereavement: Theory, Research and Intervention. New York, Cambridge University Press.

Walsh, F., 2006. Strengthening Family Resilience. New York, Guilford Press.

Worden, W., 2004. El tratamiento del duelo: asesoramiento psicológico y terapia. Barcelone, Paidós.

Yoffe, L., 2013. « Nuevas Concepciones sobre los Duelos por pérdidas de seres queridos », Avances en Psicología: Revista de la Facultad de Psicología y Humanidades, vol. 21, $\mathrm{n}^{\circ} 2$, p. 129-153.

\footnotetext{
${ }^{1}$ Les auteurs souhaitent remercier chaleureusement les personnes migrantes qui ont participé à la recherche, les refuges qui leur ont ouvert leurs portes, ainsi que Joseba Achotegui pour ses recommandations au moment de commencer cette étude.

${ }^{2}$ Ces refuges, généralement administrés par des églises, sont présents sur toutes les routes qu'utilisent traditionnellement les migrants au Mexique. Ils offrent un hébergement temporaire sûr pour les personnes en processus de migration.

${ }^{3}$ Le culte à la Santa Muerte est un culte d'origine populaire qui s'est développé durant les dernières années et qui est considéré par ses adeptes comme la réponse à leurs besoins et à leurs problèmes qu'ils n'ont pas trouvée dans leur religion d’origine. Il est généralement associé aux actes délictueux de certains de ses dévots, bien que la majeure partie de ses adeptes provienne de diverses classes sociales (Reyes 2011).

${ }^{4}$ Selon des données d'Insight Crime, le Salvador présentait le taux d'homicide le plus élevé du monde en 2015 (103 pour 100000 habitants), tandis que le Honduras figurait aussi au plus haut de ce sinistre classement, avec 57 homicides pour 100000 habitants (Insight Crime 2016).

${ }^{5}$ Le Seguro Popular est un programme de couverture universelle de santé que le gouvernement mexicain a lancé en 2001 et qui a été étendu en 2015 à toutes les personnes présentes sur le territoire mexicain, indépendamment de leur nationalité ou de leur statut migratoire.
} 